This is a non-peer reviewed preprint submitted to EarthArXiv

It was submitted on Feb. 11, 2019 to Geosphere

\title{
RSFit3000: A MATLAB GUI-Based Program for Determining Rate and State Frictional Parameters from Experimental Data
}

\author{
Rob M. Skarbek ${ }^{* 1}$ and Heather M. Savage ${ }^{1}$ \\ ${ }^{1}$ Lamont-Doherty Earth Observatory, Columbia University
}

\section{Abstract}

We present a MATLAB, graphical user interface (GUI) software package for analyzing rate and state friction experiments. Called RSFit3000, the software allows users to easily constrain frictional parameters by fitting velocity step and slide-hold-slide events using the aging and slip law forms for state variable evolution. RSFit3000 includes features for removing strain hardening/weakening trends from the data, and provides options for using two state variables, applying a weighting function, and treating stiffness as a fitting parameter. Completed fits are conveniently saved in MATLAB structure arrays that contain fitted parameter values with their error intervals, as well as all information required to reproduce a given fit. The GUI makes the program simple to use, as all fitting tasks are completed via interaction with the interface. Here we describe how to use the software, and illustrate its flexibility and utility by analyzing two sets of synthetic friction data, as well as some previously published experimental data. Although descriptions of rate and state friction fitting routines have been published in the past, RSFit3000 marks the first time a software package for analyzing friction experiments has been described in the literature.

\section{Introduction}

Rock friction experiments have provided the basis for the rate and state friction equations, which represent the current best description of sliding friction available to the Earth science community. Although rate and state friction theory is by far mostly used to describe various aspects of fault slip (e.g. Marone, 1998; Lapusta et al., 2000; Rubin, 2008), it also successfully describe glacier sliding (e.g. Zoet et al., 2013; McCarthy et al., 2017), and landslides (Handwerger et al., 2016). The equations describe the evolution of friction coefficient as a function of sliding velocity, and an internal state variable that can be thought of as representing the average lifetime of frictional contacts (Scholz, 1998).

The rate and state equations are empirical, and so regardless of any physical interpretations that have been attached to them (e.g. Rice et al., 2001), it is necessary to carry out a numerical inversion to determine values of the constitutive parameters for any given experiment. The details of numerical routines for carrying out such inversions have been published by Reinen and Weeks (1993), Blanpied et al. (1998), and Noda et al. (2009). However, none of these works includes any code and at present, a number of undocumented codes, that have been distributed via informal sharing, are currently in use in the experimental rock friction community.

*rskarbek@ldeo.columbia.edu 
Here we describe a MATLAB graphical user interface-based program called RSFit3000, that provides an easy to use method for determining rate and state frictional parameters from experimental data. The code is available at https://github.com/rmskarbek/RSFit3000. RSFit3000 is capable of fitting velocity step events, as well as slide-hold-slide (SHS) events, using both the aging and slip law formulations for state variable evolution. It can also perform fits using two state variables, and includes an options for treating the stiffness as a fitting parameter, and for producing weighted fits. A detailed User Guide is included in the Supplementary Materials. Here we describe the main features of the program and the work flow involved in obtaining fits to data. Finally, we suggest some best practices for fitting and reporting rate and state friction variables.

\section{Background}

The rate and state friction equations are empirical relationships that describe how the friction coefficient $\mu$ of sliding rock surfaces, or gouge layers, evolves with the sliding velocity $v$ and one or more internal state variables $\theta_{i}$ (Marone, 1998). The friction coefficient is usually written as

$$
\mu=\mu_{0}+a \ln \left(\frac{v}{v_{0}}\right)+\sum_{i} b_{i} \ln \left(\frac{v_{0} \theta_{i}}{d_{c i}}\right),
$$

where $\mu_{0}$ is a reference friction coefficient that prevails for steady-state sliding at reference velocity $v_{0}, a$ and $b_{i}$ are constitutive parameters that determine how the steady-state value of $\mu$ evolves with sliding velocity, and $d_{c i}$ is a critical slip distance that is related to the amount of slip needed to attain a new steady-state after changes in sliding velocity.

A second equation is needed to describe the evolution of the state variable. By far, the two most widely used equations are the aging law

$$
\frac{\partial \theta_{i}}{\partial t}=1-\frac{v \theta_{i}}{d_{c i}}
$$

and the slip law

$$
\frac{\partial \theta_{i}}{\partial t}=-\frac{v \theta_{i}}{d_{c i}} \ln \left(\frac{v \theta_{i}}{d_{c i}}\right)
$$

Other equations have also been proposed (e.g. Marone, 1998; Nagata et al., 2012), but are not in common use for experimental data, and we note that recent experimental and theoretical work has shown that the slip law is capable of describing a larger range of experimental results than the aging law (Bhattacharya et al., 2015). RSFit3000 makes use of both equations (2) and (3), with one or two state variables $\theta_{i}$.

There are three unknowns $(\mu, v, \theta)$ present in equation (1) along with equation (2) or (3), and so a third equation is needed. For the purposes of studying experimental friction data, Hooke's law applied to a single degree or freedom spring-slider is always used to approximate an elastic response (Figure 1A). In terms of the friction coefficient the relation is

$$
\frac{\partial \mu}{\partial t}=k\left(v_{l}-v\right)
$$

where $k$ is the elastic stiffness with units $1 /$ length, and $v_{l}$ is the velocity of the load point. Inertia is ignored, and the slider block is assumed to be rigid, so that all elastic effects associated with deformation of the sliding surface and testing machine are represented by the spring stiffness (Marone, 1998). Use of equation (4) also comes with an assumption that the normal stress $\sigma$ on the sliding 

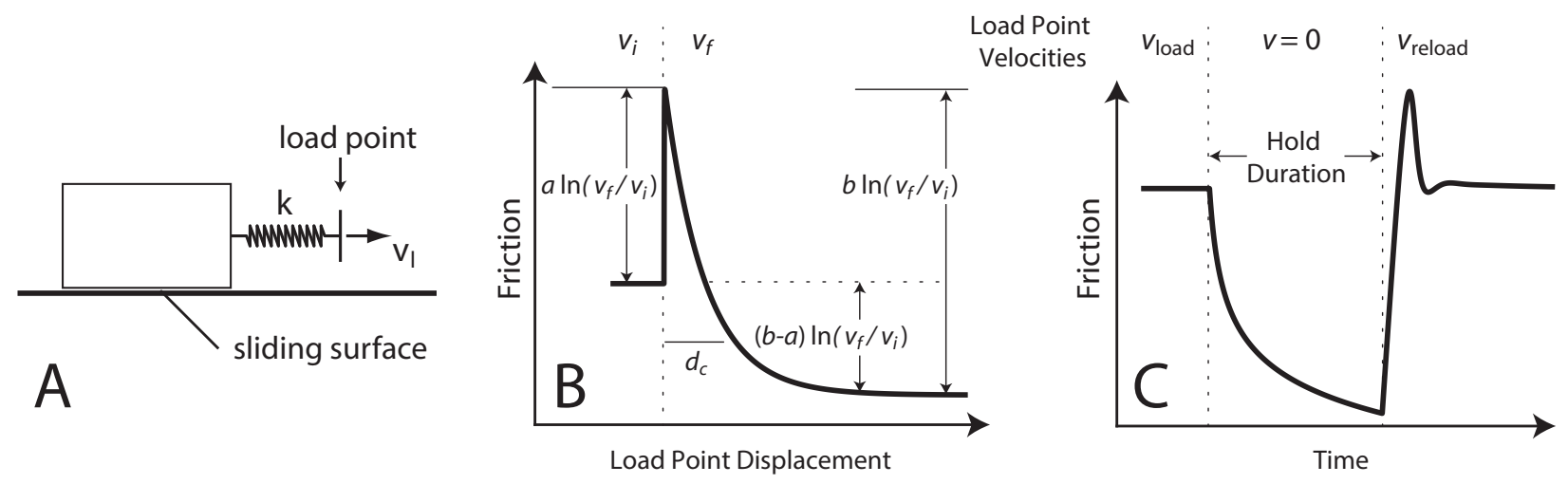

Figure 1: (A) Conceptual diagram of the spring-slider model. A rigid block is pulled by a load point via a spring of stiffness $k$, and slides along a frictional surface. (B) Illustration of a velocity up-step event. The load point velocity changes abruptly from $v_{i}$ to $v_{f}$. (C) Illustration of a slide-hold-slide event. The load point is set to zero from an initial velocity $v_{\text {load }}$, and after a specified time is set moving again at a velocity $v_{\text {reload }}$.

surface is constant, so that multiplying equation (4) by $\sigma$ results in an equation for the rate of change of the shear stress on the sliding surface with stiffness in units of stress/length.

RSFit3000 is suitable for the analysis of any friction experiment where the normal stress remains constant. It does not take into account any geometrical or frictional effects that can occur as a result of changing normal stress, such as Linker-Dieterich effects (e.g. Linker and Dieterich, 1992), or experiments conducted on sliding surfaces that are not orthogonal to the loading axes (e.g. He et al., 1998).

Experimental measurements of rock friction are commonly made using two types of imposed friction events: velocity steps, and slide-hold-slides (SHS). In a velocity step, the load point is set in motion at a constant rate $v_{i}$ for a sufficient displacement that a steady friction coefficient is achieved. The sliding rate of the load point is then changed as quickly as possible to a new value $v_{f}$. The change in sliding rate induces a "direct effect" and an "evolution effect" in the measured value of the friction coefficient (Figure 1B). We describe these effects for a velocity increase $\left(v_{f}>v_{i}\right)$, the effects are reversed if $v_{f}<v_{i}$. Immediately after the change in sliding rate, the friction coefficient will increase to a local maximum, changing by an amount $a \ln \left(v_{f} / v_{i}\right)$; this is the direct effect. After reaching a peak value, the friction coefficient will decay to a new steady state value as sliding continues, changing by an amount $-b \ln \left(v_{f} / v_{i}\right)$ from the peak value; this is the evolution effect. If $a-\sum_{i} b_{i}<0$, the steady state friction value at $v_{f}$ will be less than that at $v_{i}$, and vice versa if $a-\sum_{i} b_{i}>0$.

A SHS event also initiates at a steady state value of friction at a load point velocity $v_{\text {load }}$. Instead of changing the load point velocity, it is stopped altogether for some amount of time (the hold), and then started again at a new value $v_{\text {reload, }}$ usually, but not necessarily, equal to the previous sliding rate. During the hold period, the friction coefficient will decay logarithmically with time. When sliding is re-initiated, the friction coefficient will increase rapidly to a peak value, and then decay to a new steady state (Figure 1C).

Due to the empirical nature of equations $(1)-(3)$, the frictional parameters $a, b_{i}$, and $d_{c i}$ must be determined by conducting forward modeling fits to experimental friction events. Additionally, since a simple spring-slider model, equation (4), is used to represent the combined elastic response of the testing machine (which depends on its geometry and materials) and the sample (which is likely evolving throughout a given experiment), the stiffness $k$ should also be determined through 
the fitting procedure as well. The value of $\mu_{0}$ can also be used as a fitting parameter, but is generally well constrained through direct measurement. Reinen and Weeks (1993), Blanpied et al. (1998), and Noda et al. (2009) all provided descriptions of techniques for fitting equations (1) - (4) to experimental data. In developing RSFit3000, we made use of a combination of techniques from each of these papers, although our methods are perhaps most similar to those of Noda et al. (2009).

\section{Methods}

RSFit3000 is built around a nonlinear least squares fitting routine that uses the LevenbergMarquardt method (e.g. Noda et al., 2009). The routine makes use of the built in MATLAB function lsqnonlin, which is part of the MATLAB Optimization Toolbox. The program will not function properly on MATLAB installations that do not have this toolbox. We give a brief description of the algorithm, detailed descriptions of the Levenberg-Marquardt method can be found in Levenberg (1944); Marquardt (1963), and Moré (1978).

Given a set of $N$ experimental measurements of friction coefficient $\mu_{\mathrm{obs}}\left(t_{i}\right)$ at times $t_{i}(i=1$, $\ldots, N)$, we seek a vector $\xi_{f}$ of parameter values $\left(a, b_{1}, d_{c 1}\right.$, and optionally $\mu_{0}, k, b_{2}$, or $\left.d_{c 2}\right)$ that yields predicted friction coefficient values $\mu_{\text {pred }}\left(t_{i}\right)$ such that the sum of the squares of an objective function $F\left(t_{i} ; \xi\right)=w_{i}\left|\mu_{\mathrm{obs}}\left(t_{i}\right)-\mu_{\mathrm{pred}}\left(t_{i} ; \xi_{f}\right)\right|$ is minimized, where $w_{i}$ is a vector of weight values generally set equal to unity. Provided with a vector $\xi_{0}$ of initial parameter value guesses, the Levenberg-Marquardt method computes updates $\Delta \xi$ to $\xi_{0}$ by solving the set of linearized equations

$$
\left[J(\xi)^{T} J(\xi)+\lambda I\right] \Delta \xi=J(\xi)^{T} F(\xi),
$$

where $J(\xi)$ is the $N$-by- $M$ Jacobian matrix of $F(\xi), M$ is the number of parameters in $\xi, I$ is the $N$-by- $M$ identity matrix, and $\lambda$ is a scalar damping parameter that controls the magnitude and direction of $\Delta \xi$. The GUI also provides an option to weight the friction data, similar to Reinen and Weeks (1993) and Blanpied et al. (1998), so that the weight vector takes on values greater than unity at user specified locations in the data. More description of the weighting procedure is provided in the next section.

When the fitting routine is started, it computes $\mu_{\text {pred }}\left(t_{i} ; \xi_{0}\right)$ by numerically simulating the experimental conditions using equations (1), (2) or (3), and (4). Simulations are carried out using the built-in MATLAB function ode45, which is an explicit Runge-Kutta based solver with an adaptive time step (Shampine and Reichelt, 1997; Ashino et al., 2000). The simulation is re-run every time the parameter values are updated.

The fitting routine updates the parameter values vector until one of several stopping criteria is met. Figure 2 schematically illustrates how the stopping criteria are evaluated. The routine will stop successfully if any of three criteria are met: 1) a local minimum in the objective function is found, 2) the relative sum of squares of the objective function (vertical distance between Points $\mathrm{A}$ and $\mathrm{B}$ ) changes by less than a defined tolerance, or 3) the norm of the step size (horizontal distance between Points A and B), changes by less than a defined tolerance. The routine will stop unsuccessfully if the number of function evaluations or solver iterations exceed defined limits, or if the current parameter values produce unstable, stick-slip events. The tolerance values and function evaluation and iteration limits can all be changed by the user (see User Guide for details), but we have found that using the default values works very well. The stopping criteria are part of lsqnonlin, and more information in tolerances and stopping criteria in general can be found in the MATLAB documentation. The routine generally only stops unsuccessfully when it is given a bad set of initial parameter values guesses $\xi_{0}$. In the next section we describe how to avoid such an outcome. 


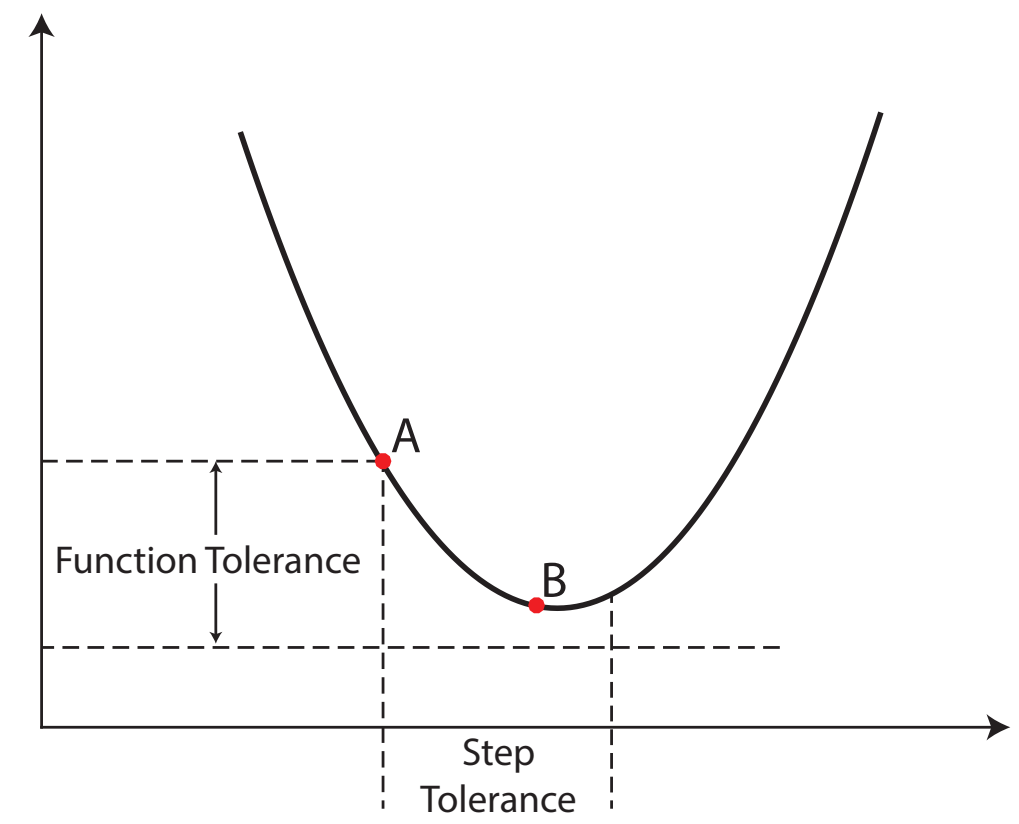

Figure 2: Schematic figure showing how the function tolerance and step tolerance stopping criteria are met. Adapted from a figure in the MATLAB help documentation. A solver iteration from Point A to Point B would satisfy both the function and step tolerance stopping criteria.

Upon successful completion of the fitting routine, lsqnonlin will output the optimized parameter values $\xi_{f}$, as well as the Jacobian matrix $J$ and the residuals $r_{i}=F\left(t_{i} ; \xi_{f}\right)$. We then calculate the covariance matrix $C$ according to

$$
C=\frac{\|r\|^{2}\left(J^{\mathrm{T}} J\right)^{-1}}{N-M}
$$

where $\|r\|=\sqrt{\sum_{i=1}^{N} r_{i}^{2}}$ is the $L_{2}$ norm of the residuals (Menke, 2018). We note that $s_{r}^{2}=\|r\|^{2} /(N-$ $M)$ is the variance of the residuals. We report errors to the fitted parameter values as $\pm 2 s$, where $s$ is the standard deviation, computed as the square root of a parameter's covariance with itself (i.e. square root of the diagonals in the covariance matrix). There can be a high degree of correlation between the frictional parameters, particularly $a$ and $b_{i}$ (e.g. Reinen and Weeks, 1993; Blanpied et al., 1998; Noda et al., 2009). The value of $a-\sum_{i} b_{i}$ is especially important, and the standard deviation $s_{a-b}$ for this combined parameter is computed using

$$
s_{a-b}^{2}=s_{a}^{2}+\sum_{i} s_{b_{i}}^{2}+2\left(C_{b_{1}, b_{2}}-\sum_{i} C_{a, b_{i}}\right),
$$

where $C_{b_{1}, b_{2}}$ is the covariance between $b_{1}$ and $b_{2}$, if it exists, and $C_{a, b_{i}}$ is the covariance between $a$ and $b_{i}$.

Finally, as a measure of the goodness of the fit provided by $\xi_{f}$, we compute the coefficient of determination $R^{2}$ as

$$
R^{2}=1-\sum_{i=1}^{N}\left[\frac{\mu_{\mathrm{obs}}\left(t_{i}\right)-\mu_{\mathrm{pred}}\left(t_{i} ; \xi_{f}\right)}{\mu_{\mathrm{obs}}\left(t_{i}\right)-\bar{\mu}_{\mathrm{obs}}}\right]^{2},
$$

where $\bar{\mu}_{\text {obs }}$ is the mean of the observed friction values. Following equation (8), $R^{2}$ takes on values between 0 and 1 , with $R^{2}=1$ corresponding to a perfect match between $\mu_{\text {obs }}\left(t_{i}\right)$ and $\mu_{\text {pred }}\left(t_{i} ; \xi_{f}\right)$. 


\section{Work Flow}

In this section we summarize the steps needed to produce fits and describe some of the mathematical procedures used in RSFit3000. The following data are required to perform fits: friction coefficient, load point displacement, normal stress, and time. Each of these data sets must be loaded into the MATLAB workspace as vectors with identical sizes, and entered into the Experimental Data Panel (3A). Once these data are input to the program, initially identical plots of friction coefficient against load point displacement appear in the Static and Windowing Axes (Figure 3B and 3C). To continue, the zoom tool is used to isolate an event of interest in the Windowing Axes, such as a single velocity step or SHS event. A red box will appear on the Static Axes showing the location of the windowed data within the entire data set.

Next, detrending is performed in the Windowing Axes if necessary. In real experimental friction data, there is often a global strain weakening or hardening trend. In other words, for sliding at a steady load point velocity, the friction coefficient decreases (weakening) or increases (hardening) at an approximately constant rate. The common practice is to remove the global trend before fitting the data with the RAS equations (Lockner et al., 1986; Tullis and Weeks, 1986; Marone et al., 1990; Chester, 1994; Beeler et al., 1996; Blanpied et al., 1998). Detrending is performed in RSFit3000 by fitting a line to selected portions of the experimental data that display a weakening or hardening trend, and using the slope of the fitted line to remove the trend (Figure 3D).

To detrend data, the user selects two points $\left(\delta_{1}, \mu_{1}\right)$ and $\left(\delta_{2}, \mu_{2}\right)$ on the windowed data and the program fits a line to the data between these points, where $\delta$ is the load point displacement. Then the user selects a reference point $\left(\delta_{r}, \mu_{r}\right)$ on the data and the program removes the trend defined by the fitted line, relative to the reference point according to the equation $\mu^{\prime}\left(t_{i}\right)=\mu\left(t_{i}\right)+m\left[\delta_{r}-\delta\left(t_{i}\right)\right]$, where $m$ is the slope of the line between points $\left(\delta_{1}, \mu_{1}\right)$ and $\left(\delta_{2}, \mu_{2}\right)$, and $\mu\left(t_{i}\right), \delta\left(t_{i}\right)$ denote the friction coefficient and load point displacement data that are visible in the Windowing Axes.

After detrending, the detrended and windowed data will appear in the Fitting Axes (Figure 3E). The load point displacement data in the Fitting Axes will be referenced to zero at the beginning of the windowed event. The proper event type (velocity step, or SHS) must be selected in the Event Type Panel (Figure 3F). There are four options available for performing a fit: 1) use of a weighting function, use of 2) $\mu_{0}$ or 3) stiffness $k$ as fitting parameters, and 4) use of two state variables (Figure $3 \mathrm{G}$ ). Any of these options may be combined with one another. The user may also select whether to use both, or one of the state evolution laws.

We implement a weight function similar to those described by Reinen and Weeks (1993) and Blanpied et al. (1998). When use of the weight function is desired, the user selects the appropriate option, then sets a value for the exponent $p$ in the Weight Parameters Window (Figure 3H), and clicks the Set Weight Location Button. The weight location is set by selecting a data point in the Fitting Axes. Once selected, the corresponding values of load point displacement and friction coefficient will appear in the Weight Parameters Window, and the program will generate a plot of the resulting weight values against load point displacement.

The weight values $w_{i}$ are calculated according to

$$
w_{i}=\left\{\begin{array}{cl}
1 & i<i_{w} \\
\left(\frac{N}{j}\right)^{p} & i \geq i_{w}
\end{array}\right.
$$

where $i=1, \ldots, N$, and $N$ is the total number of windowed data points, $j$ is an index that runs from 1 to $N-i_{w}$, and $i_{w}$ is the index of the selected weight location (taken from the set of indices $i$, rather than $j$ ). When $p=2$, equation (9) appears to be identical to the weight function used by Blanpied et al. (1998). 


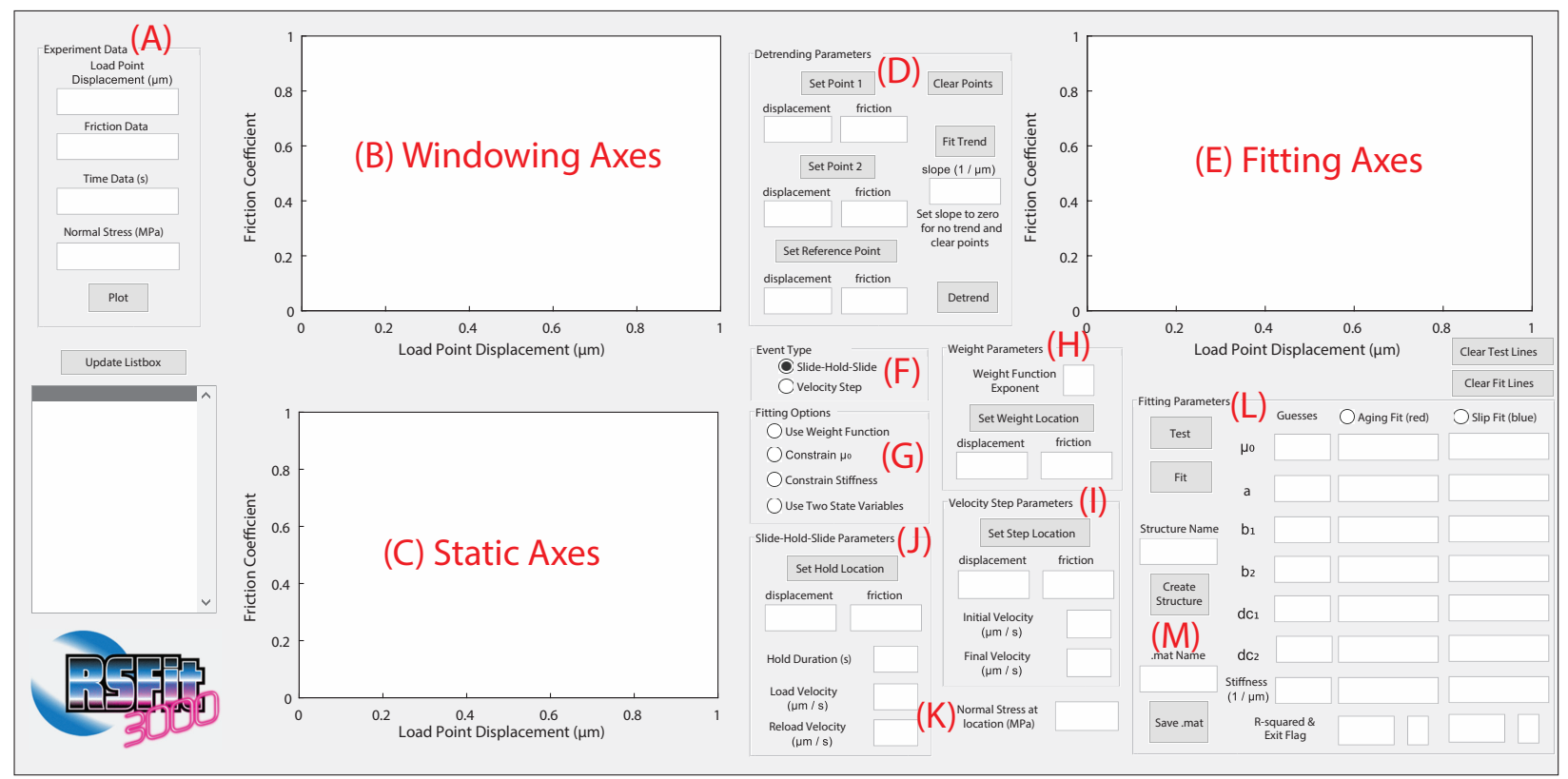

Figure 3: Layout of the MATLAB graphical user interface for RSFit3000, with labeled features. Detailed information on use of the GUI can be found in the supplementary User Guide. (A) Experimental Data Panel, (B) Windowing Axes, (C) Static Axes, (D) Detrending Parameters Panel, (E) Fitting Axes, (F) Event Type Options, (G) Fitting Options, (H) Weight Parameters Panel, (I) Velocity Step Parameters Panel, (J) Slide-Hold-Slide Parameters Panel, (K) Normal Stress Display, (L) Fitting Parameters Panel, (M) Create Structure Button.

The effects of using a weight function following equation (9) have been discussed by Reinen and Weeks (1993), who use a weight function, and also by Noda et al. (2009), who do not use a weight function. Reinen and Weeks (1993) and later Blanpied et al. (1998)'s motivation for using a weight function is that the fitted values of the rate and state parameters depend mostly on the evolution of the friction values after the peak value is reached following a velocity up-step (i.e. on the evolution effect), whereas the rise in friction values to the peak itself (i.e. the direct effect) is highly influenced by the value of the stiffness $k$. As such, neither Reinen and Weeks (1993) or Blanpied et al. (1998) treat the stiffness as a fitting parameter. Instead they give $k$ a constant value, and argue that the friction values recorded after the peak value should be weighted more heavily than other portions of a velocity up-step event. Thus they use weight functions similar to equation (9) with the weight location set at the peak friction value. Alternatively, Noda et al. (2009) do treat the stiffness as a fitting parameter, and point out that using a weighting function in such a case is likely to increase uncertainty in the fitted value of $k$, since the portion of the up-step event that depends most on the stiffness would be weighted less than other portions of the data. We leave it to the user to decide upon the use of a weight function, but note that its use can occasionally produce better fits, even when fitting the stiffness, as illustrated in the next section.

The procedures for fitting velocity steps and SHS events are very similar. For a velocity step, the user selects the location of the step in the data on the Fitting Axes. The values of load point displacement and friction coefficient from the selected point will appear in the Velocity Step Parameters Panel (Figure 3I) and the program will generate a plot of the load point displacement against time from the windowed data, showing the location of selected step. The program will also calculate and display the load point velocity before and after the selected step location, using the 
load point displacement time series before and after the selected step (see User Guide for more details). All of these values can also be manually entered. However, manually entering velocity values that do not closely correspond to the actual velocities will have a detrimental effect on the goodness of the fit.

For a SHS event, the user selects the location of the beginning of the hold on a separate plot of friction coefficient against time that appears when the Set Hold Location Button is clicked (Figure $3 \mathrm{~J})$. This helps in choosing the correct location, as it can sometimes be difficult to properly identify the start of a hold on a plot of friction against load point displacement on the Fitting Axes. After selecting the hold location, the program will not automatically compute the remaining values in the Slide-Hold-Slide Parameters panel. The user must manually enter values for the hold duration, and the load point velocities before (Load Velocity) and after (Reload Velocity) the hold. When either a velocity step, or SHS location is selected, the normal stress at the time of the location will appear below the Velocity Step Parameters Window (Figure 3K).

To apply the fitting procedure (Figure 3L), the user selects whether to perform fits using either, or both, the aging and slip laws for state evolution. The user then enters trial values for $\mu_{0}, a, b_{1}$, $d_{c 1}, k$, and $b_{2}$ and $d_{c 2}$ if using two state variables. Values for $\mu_{0}$ and $k$ must be entered regardless of whether the options to constrain these two values are selected. After entering the trial values, clicking the Test Button will run a spring-slider simulation using the trial values, and the results will appear in the Fitting Axes. Depending on the event type, the program uses the event parameters to simulate the correct behavior. For a velocity step event, the program determines the time that the step occurred when the user selects the step location. This information, along with the initial and final load point velocities, is passed to the spring-slider program and used to simulate the proper behavior of the load point. A similar procedure is used to simulate a SHS event. Information about when the hold initiates, how long it lasts, and the load point velocities before and after the hold are passed to the spring-slider program. If the test lines do not closely overlie the data, any of the trial values can be changed to run another test. This process should be repeated until the test lines overlie the data as much as possible. Obtaining trial values in this manner is critical to obtaining good fitted parameter values.

After generating satisfactory parameter guesses, the user clicks the Fit Button, and the program carries out the nonlinear least squares fitting routine. Information regarding the number of solution attempts, and the parameter values for each attempt will be displayed in the MATLAB Command Window as the fitting routine is running. When the routine has completed, the results will appear in the Fitting Axes, and the fitted parameter values will be displayed in the Fitting Parameters Panel. Each fitted parameter value will be displayed with its $2 s$ error interval. These values, along with all of the information used to produce the fit, can be saved as a structure array in the MATLAB workspace by entering a name in the Structure Name Field and clicking the Create Structure Button (Figure 3M).

The created structure contains the load point displacement data, measured friction coefficient, detrended friction coefficient, and time values from the windowed event. It also contains the parameters that were used to detrend the friction data, as well as the initial parameter guesses, the fitted parameter values with the error intervals, the $R^{2}$ value and covariance matrix, and the predicted friction coefficient values generated by the fitting procedure. If the event was a velocity step, the structure will contain the initial and final load point velocities, the time and the normal stress at which the step occurred. If the event was a SHS, the structure will contain the load and reload velocities, the time and normal stress at which the hold initiated, and the duration of the hold. If the weight function was used, the relevant parameters will be in the structure as well. The user can window another velocity step or SHS event in the Windowing Axes and repeat the procedures described above to obtain more fits. 


\section{$6 \quad$ Examples}

In this section we present some examples that illustrate the features of RSFit3000 and techniques that can be used to fit friction events. First, we present some results from a synthetic data set. That allows us to produce events that require different techniques to obtain good fits. In contrast to real experimental data, synthetic data is generated using a defined set of parameter values, as well as a specific choice of state evolution law. However, to mimic real data, we add a significant amount of noise to our synthetic data, and the goal of our fits is not to exactly recover the original parameter values. The addition of noise allows for a more realistic demonstration of the fitting routine. Indeed fits to synthetic data that did not have added noise would always produce the original parameter values with extremely small error estimates, and be of little instructive value. Finally, we present some results from fits to experimental data previously published by Ikari et al. (2014), that illustrates the effects that detrending can have on fit results. All of the information needed to reproduce the fits shown in this paper can be found in the Supplementary Materials.

\subsection{Synthetic Data}

We generated two synthetic experimental data sets by running a spring-slider simulation using the slip law. The synthetic data and the original parameter values are shown in Figure 4 . The data points were generated at $100 \mathrm{~Hz}$. The data shown in Figure 4A uses a single state variable, and that shown in Figure 4B uses two state variables. Both sets were generated using an identical load point history, consisting of four events: 1) a velocity up-step from $1 \mu \mathrm{m} / \mathrm{s}$ to $10 \mu \mathrm{m} / \mathrm{s}$; 2) a velocity down-step from $10 \mu \mathrm{m} / \mathrm{s}$ to $1 \mu \mathrm{m} / \mathrm{s}$; 3) a SHS of $10 \mathrm{~s}$ duration with identical load and reload velocities of $1 \mu \mathrm{m}$; and 4) a SHS of $1000 \mathrm{~s}$ duration with a load velocity of $1 \mu \mathrm{m} / \mathrm{s}$ and a reload velocity of $10 \mu \mathrm{m} / \mathrm{s}$. Random Gaussian noise was added to both data sets (e.g. Noda et al., 2009) using the MATLAB function randn in the equation $\mu=\nu \operatorname{randn}(N, 1)+\mu_{s}$, where $\mu_{s}$ is the original synthetic value of the friction coefficient, $N$ is the number of samples, and $\nu=0.005$ is the variance of the added noise signal.

The fits to the events from both data sets are shown in Figures 5 and 6, along with the fitted parameter values and their error intervals. The fits demonstrate the flexibility of the features included in RSFit3000. Frequently, it's possible to fit an event with both state evolution laws using a single set of initial parameter guesses $\xi_{0}$. Then the aging and slip law fits can be generated simultaneously by selecting both laws in the Fitting Parameters Window. We used this procedure to fit the velocity up-step (Figures 5A and 6A) and the first SHS event (Figures 5C and 6C) for both the single and two state variable data. Sometimes a set of initial parameter guesses will generate a good fit using one state evolution law, but not the other; this was the case for the velocity down-step in the one state variable data (Figure 5B). To fit that event, a different set of values for $\xi_{0}$ can be used for each state evolution law, and the fits generated individually by selecting the desired law.

It may also occur, especially when using synthetic data, that the routine cannot produce a fit for one of the evolution laws; this occurred for the second SHS in both data sets (Figures 5D and 6D), as well as the velocity down-step in the two state variable data. If both state evolution laws are selected, the fitting routine will attempt the produce an aging law fit first. If the aging law attempt fails, then the routine will stop and no fitting results will be displayed on the Fitting Axes or in the Fitting Parameters Window for either law. If the slip law attempt fails, results for a successful aging law attempt will still be displayed. The law that failed to produce a fit can be deselected, and the routine run successfully for the other law. In the case of the synthetic data, we could only successfully generate fits for these specific events while using the slip law.

The first SHS event in the two state variable data illustrates the sometimes ambiguous nature 


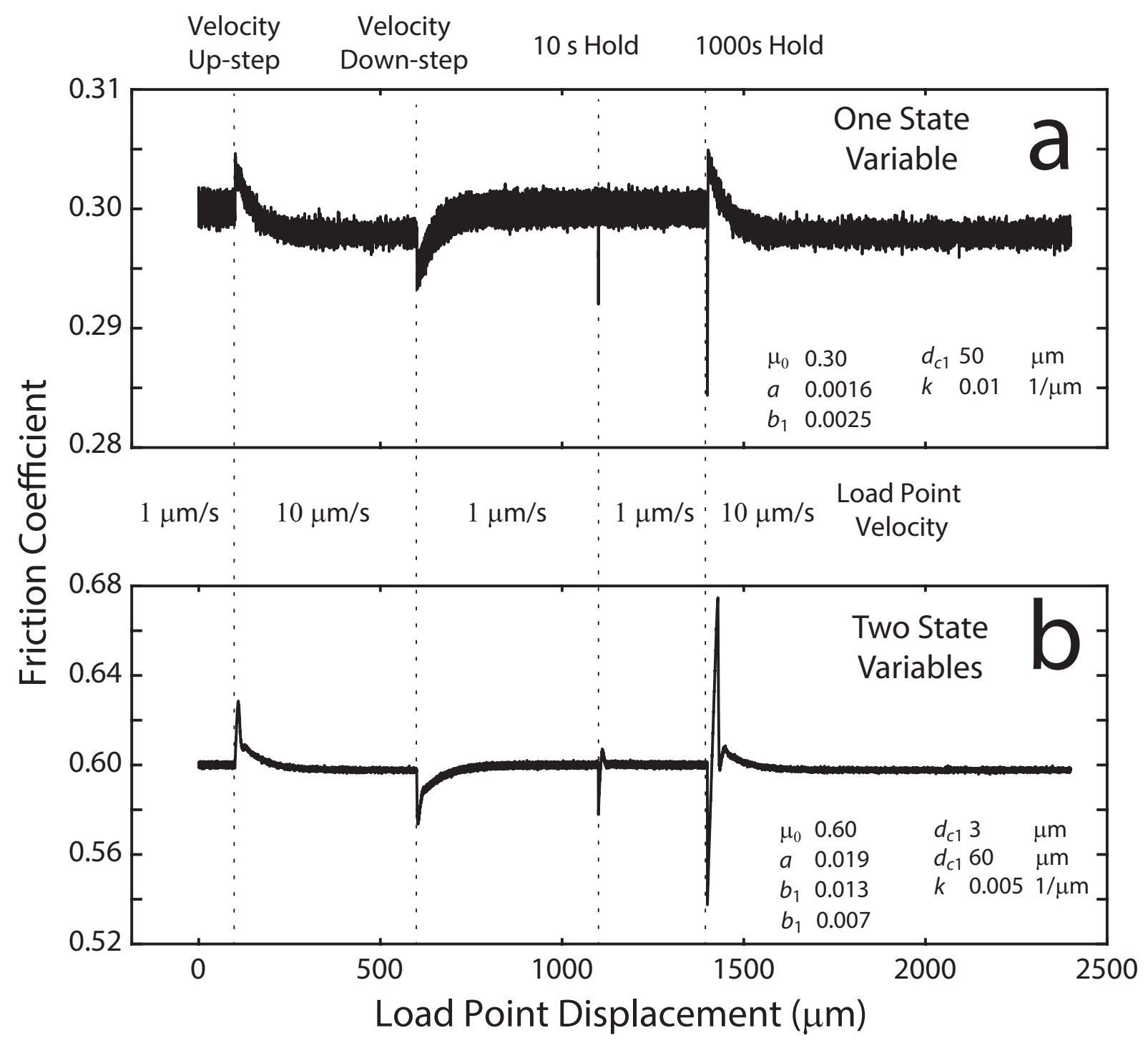

Figure 4: Synthetic friction data generated using (A) a single state variable and (B) two state variables. The parameter values used to generate each data set are shown on the figures. Both sets were generated using an identical load point history consisting of a velocity up-step, a velocity down-step, a 1 s SHS event, and finally a 1000 s SHS event. The locations of each event are labeled at the top of the figure, and the load point velocities are shown in between the two panels.

of the rate and state friction equations. For this event we can obtain fits using a single state variable (Figure 6E) that is just as good as fits obtained using two state variables (Figure 6C). Hence, without prior knowledge of how the data were generated, one would be unable to properly determine the state evolution behavior from examining this particular SHS event.

The second SHS event in the two state variable data demonstrates a case where using the weight function can produce a better fit (Figure 6D). Both the weighted and unweighted fits for this event were produced using the slip law. The unweighted fit visibly overshoots the peak friction that is attained at reloading after the hold, and also does not quite capture the slight oscillation that occurs before friction attains a new steady state value. The clear visual offset of the unweighted fit motivates the use of the weight function. For this event, we applied the weight function by choosing 

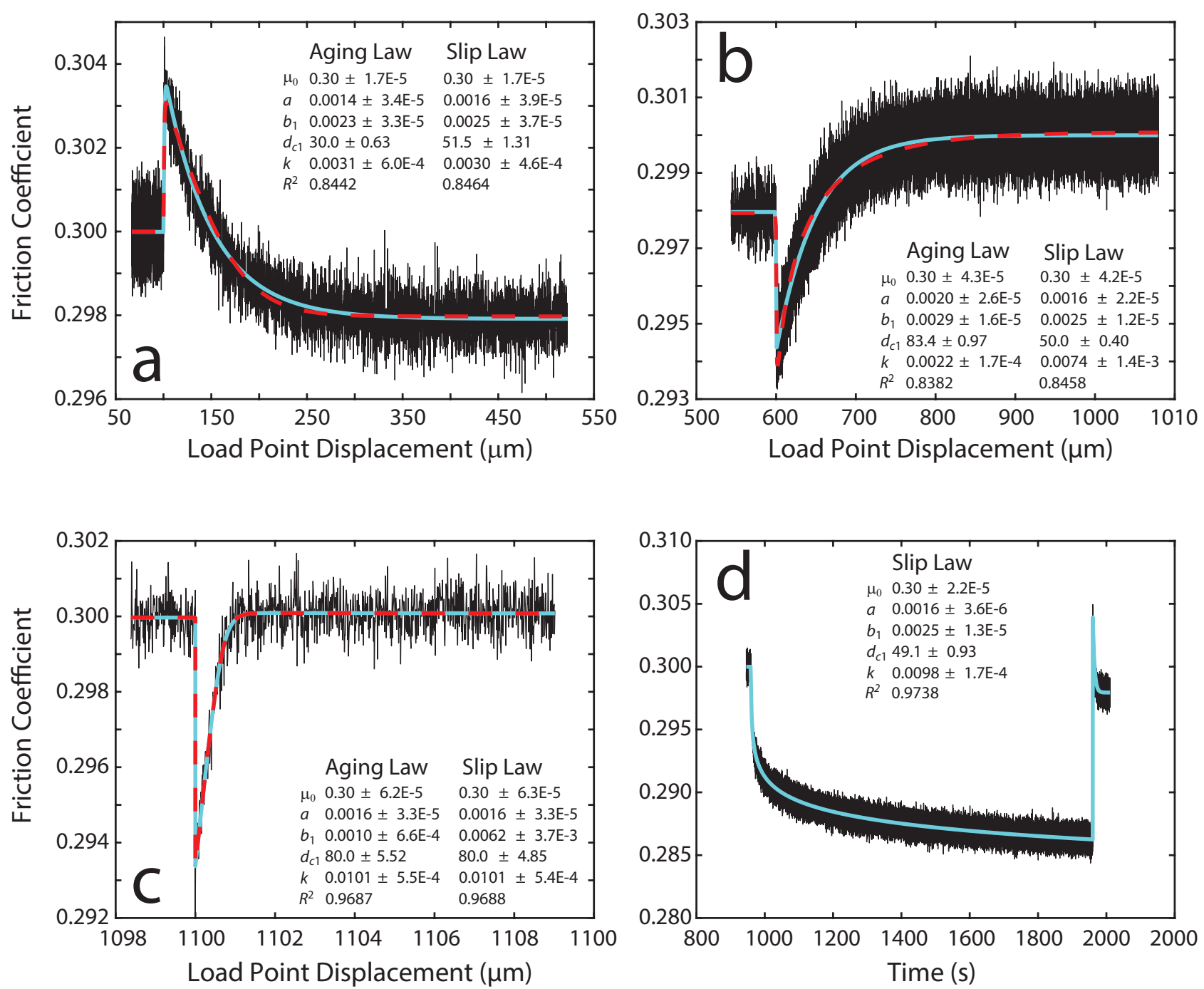

Figure 5: Fitting results for the single state variable synthetic data. (A) velocity up-step, (B) velocity down-step, (C) first SHS, (D) second SHS. Solid cyan lines show slip law results, and dashed red lines show aging law results. For each event, the fitted parameter values with their $2 s$ error intervals are shown, along with the $R^{2}$ value for the fit. Note that the second SHS is plotted against time, rather than load point displacement, for ease of viewing. Units for $d_{c 1}$, are $\mu \mathrm{m}$, units for stiffnesses $k$ are $1 / \mu \mathrm{m}$.

an exponent of $p=0.2$ and setting the weight location at the peak friction value. The resulting weight values are shown in Figure $6 \mathrm{~F}$. When using the weight function, we find that it is best to start with a small exponent (e.g. $p=0$ corresponds to an unweighted fit), and slowly increase the value until the desired effect is achieved. The weighted fit does a much better job at reproducing the entire frictional response during the SHS event. We note that it is entirely possible that using a different set of parameter guesses could have generated a good fit without use of the weighting function. The ability to apply a weight function to the fitting procedure increases the utility of RSFit3000. 

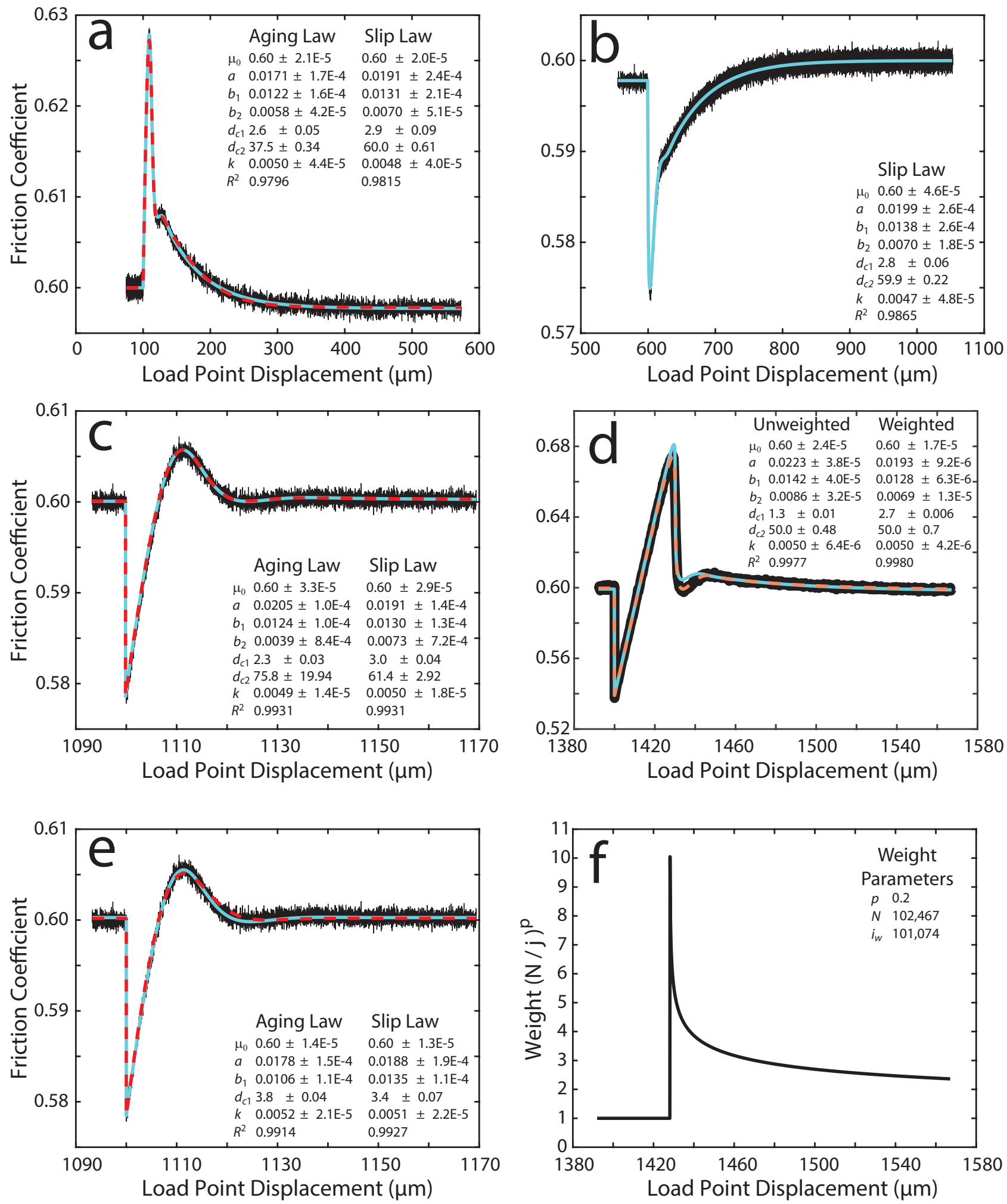

Figure 6: Fitting results for the two state variable synthetic data. (A) velocity up-step, (B) velocity down-step, (C) first SHS with two state variable fits, (D) second SHS, (E) first SHS with single state variable fits, (F) weight function values for the second SHS. Solid cyan lines show slip law results, and dashed red lines show aging law results. For panel (D) the dashed orange line shows weighted slip law results, the cyan line shows unweighted results. For each event, the fitted parameter values with their $2 s$ error intervals are shown, along with the $R^{2}$ value for the fit. Units for $d_{c 1}, d_{c 2}$ are $\mu \mathrm{m}$, units for stiffnesses $k$ are $1 / \mu \mathrm{m}$. 

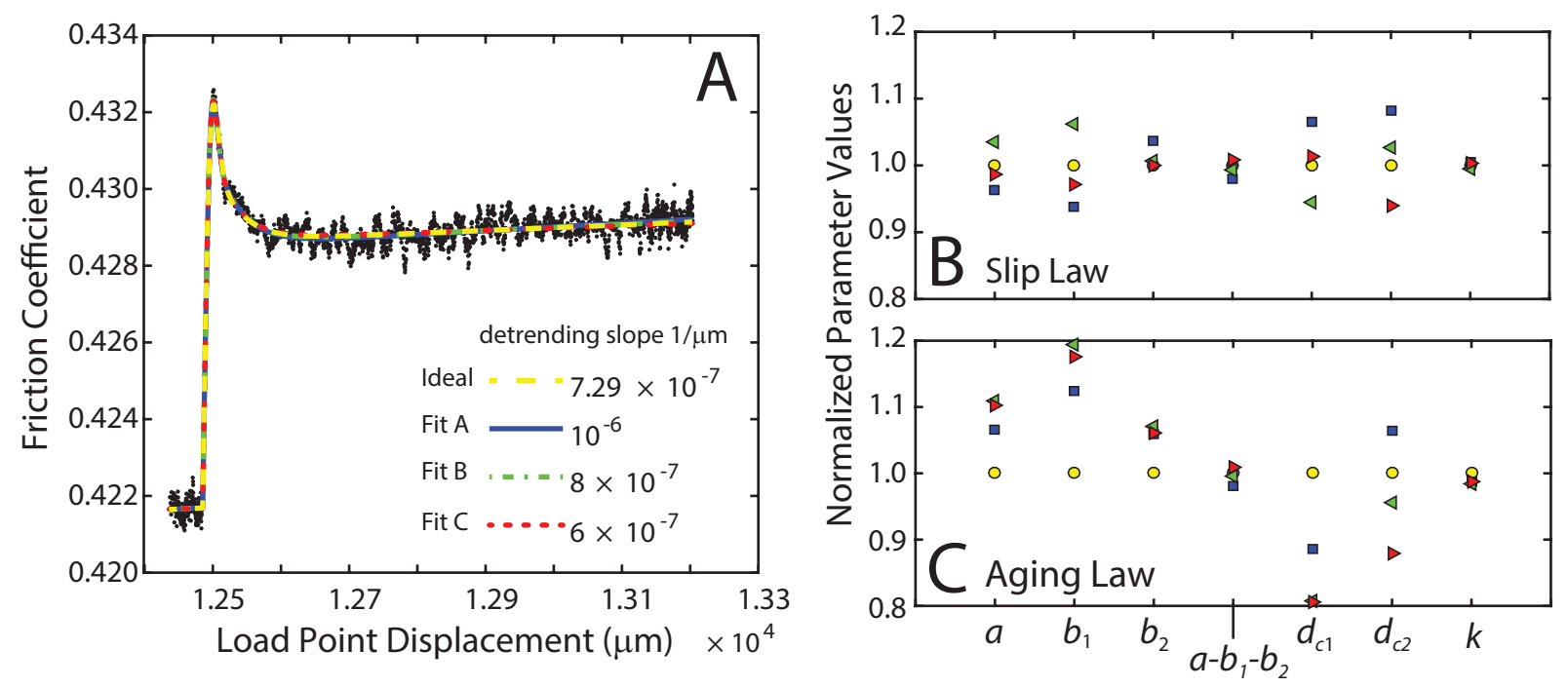

Figure 7: (A) An experimental velocity up-step from Ikari et al. (2014). Load point velocity changes from $10 \mu \mathrm{m} / \mathrm{s}$ to $30 \mu \mathrm{m} / \mathrm{s}$. Four slip law fits are shown, the only difference between them being the value of the detrending slope, as shown in the legend. (B) and (C) Parameter values from the fits shown in panel (A) and from four aging law fits that used the same detrending slope values as the slip law fits. In both cases, the parameter values are normalized by the values determined for the Ideal Fit. Symbol colors correspond to detrending slope values shown in panel (A).

\subsection{Experimental Data}

In this section we use experimental data previously published by Ikari et al. (2014) to demonstrate how the detrending procedure can influence the fitted parameter values. We use friction data that was measured on powdered gouge material collected from the Alpine Fault in New Zealand during the Deep Fault Drilling Project (Ikari et al., 2014). The measurements were conducted using a double-direct shear apparatus at Penn State University.

A velocity up-step from $10 \mu \mathrm{m} / \mathrm{s}$ to $30 \mu \mathrm{m} / \mathrm{s}$, conducted at $30 \mathrm{MPa}$ normal stress, is shown in Figure 7A, along with four fits to the data. The up-step is identical to that shown in Figure 2c of Ikari et al. (2014). Each of the fits in Figure 7A was generated in RSFit3000 using a different detrending slope, but identical detrending reference points. The fit labeled "ideal" was generated using the detrending procedure described in Section 4: we picked two points on the data to fit a detrending line, finding a slope of $m=7.29 \times 10^{-7} 1 / \mu \mathrm{m}$. The fits labeled $\mathrm{A}, \mathrm{B}$, and $\mathrm{C}$ were generated by manually entering different values for the detrending slope. Each fit was generated using the same location for the velocity step, and identical values for the parameter guesses. So, the only thing that differentiates the fits is the value of the detrending slope. Figure 7A shows fits that were generated using the slip law, but we also generated fits using the aging law. The aging law fits are visibly indistinguishable from the slip law fits, so we only show the slip law fits.

Since the detrending procedure is a subjective process, there is no "correct" fit. The four fits all have similar $R^{2}$ values, and could be said to approximately fit the data equally well. The fitted parameter values are also similar for each of the fits. Figures 7B (slip law) and 7C (aging law) show the fitted parameter values for all of the fits, normalized by the value obtained in the Ideal Fit. Note that the scatter in smallest for the quantity $a-\sum_{i} b_{i}$ for both evolution laws. Aside from this, we do not attempt to identify or explain any trends in the parameters values as a function of the detrending slope value. Rather, we emphasize that the detrending procedure does affect the fitted 
parameter values, and therefore any parameters associated with detrending should be reported when publishing fits to experimental data. RSFit3000 makes doing this convenient and easy.

\section{$7 \quad$ Best Practices}

In conclusion, we recommend a set of best practices to follow when analyzing rock friction experiments using RSFit3000. RSFit3000 is designed to enable easy reporting of all relevant information that is involved in producing a model fit to an experimental friction event. This information includes the fitted parameter values and their error intervals, the covariance matrix between the parameters, the $R^{2}$ value of the fit, parameters describing the event, parameters used to detrend the data, and parameters used to weight the data. All of this information can be saved in a MATLAB structure array when the user produces a fit. Any published fits should include these structure arrays; we also include a MATLAB script in the Supplementary Materials for writing information contained in RSFit300 structure arrays into excel files. Finally, we emphasize again that the stiffness should always be treated as a fitting parameter, due to the limitations of the single degree of freedom spring-slider model.

\section{References}

Ashino, R., M. Nagase, and R. Vaillancourt (2000), Behind and beyond the matlab ode suite, Comput. Math. Appl., 40(4), 491 - 512, doi:https://doi.org/10.1016/S0898-1221(00)00175-9.

Beeler, N. M., T. E. Tullis, M. L. Blanpied, and J. D. Weeks (1996), Frictional behavior of large displacement experimental faults, J. Geophys. Res., 101(B4), 8697-8715, doi:10.1029/96JB00411.

Bhattacharya, P., A. M. Rubin, E. Bayart, H. M. Savage, and C. Marone (2015), Critical evaluation of state evolution laws in rate and state friction: Fitting large velocity steps in simulated fault gouge with time-, slip-, and stress-dependent constitutive laws, J. Geophys. Res., 120(9), 63656385, doi:10.1002/2015JB012437.

Blanpied, M. L., C. J. Marone, D. A. Lockner, J. D. Byerlee, and D. P. King (1998), Quantitative measure of the variation in fault rheology due to fluid-rock interactions, J. Geophys. Res., 103(B5), 9691-9712, doi:10.1029/98JB00162.

Chester, F. M. (1994), Effects of temperature on friction: Constitutive equations and experiments with quartz gouge, J. Geophys. Res., 99(B4), 7247-7261, doi:10.1029/93JB03110.

Handwerger, A. L., A. W. Rempel, R. M. Skarbek, J. J. Roering, and G. Hilley (2016), Rateweakening friction characterizes both slow sliding and catastrophic failure of landslides, Proc. Natl. Acad. Sci. U.S.A., 113(37), 10,281-10,286, doi:10.1073/pnas.1607009113.

He, C., S. Ma, and J. Huang (1998), Transition between stable sliding and stick-slip due to variation in slip rate under variable normal stress condition, Geophys. Res. Lett., 25(17), 3235-3238, doi: 10.1029/98GL02518.

Ikari, M. J., B. M. Carpenter, A. J. Kopf, and C. Marone (2014), Frictional strength, ratedependence, and healing in DFDP-1 borehole samples from the Alpine Fault, New Zealand, Tectonophysics, 630, 1 -8, doi:https://doi.org/10.1016/j.tecto.2014.05.005. 
Lapusta, N., J. R. Rice, Y. Ben-Zion, and G. Zheng (2000), Elastodynamic analysis for slow tectonic loading with spontaneous rupture episodes on faults with rate-and-state dependent friction, $J$. Geophys. Res., 105(B10), 23,765-23,789, doi:10.1029/2000JB900250.

Levenberg, K. (1944), A method for the solution of certain non-linear problems in least squares, $Q$. Appl. Math., 2(2), 164-168, doi:https://doi.org/10.1090/qam/10666.

Linker, M. F., and J. H. Dieterich (1992), Effects of variable normal stress on rock friction: Observations and constitutive equations, Journal of Geophysical Research: Solid Earth, 97(B4), 4923-4940, doi:10.1029/92JB00017.

Lockner, D. A., R. Summers, and J. D. Byerlee (1986), Effects of temperature and sliding rate on frictional strength of granite, Pure Appl. Geophys., 124(3), 445-469, doi:10.1007/BF00877211.

Marone, C. (1998), Laboratory-derived friction laws and their application to seismic faulting, Annu. Rev. Earth Planet. Sci., 26, 643-696, doi:10.1146/annurev.earth.26.1.643.

Marone, C., C. B. Raleigh, and C. H. Scholz (1990), Frictional behavior and constitutive modeling of simulated fault gouge, J. Geophys. Res., 95(B5), 7007-7025, doi:10.1029/JB095iB05p07007.

Marquardt, D. W. (1963), An algorithm for least-squares estimation of nonlinear parameters, $J$. Soc. Indust. Appl. Math., 11(2), 431-441.

McCarthy, C., H. Savage, and M. Nettles (2017), Temperature dependence of ice-on-rock friction at realistic glacier conditions, Phil. Trans. R. Soc. A, 375(2086), 20150,348, doi: 10.1098/rsta.2015.0348.

Menke, W. (2018), Geophysical data analysis: Discrete inverse theory, Academic press.

Moré, J. J. (1978), The levenberg-marquardt algorithm: Implementation and theory, in Numerical Analysis, pp. 105-116, Springer.

Nagata, K., M. Nakatani, and S. Yoshida (2012), A revised rate- and state-dependent friction law obtained by constraining constitutive and evolution laws separately with laboratory data, $J$. Geophys. Res., 117(B2), doi:10.1029/2011JB008818.

Noda, H., E. M. Dunham, and J. R. Rice (2009), Earthquake ruptures with thermal weakening and the operation of major faults at low overall stress levels, J. Geophys. Res., 114, B07302, doi:10.1029/2008JB006143.

Reinen, L. A., and J. D. Weeks (1993), Determination of rock friction constitutive parameters using an iterative least squares inversion method, J. Geophy. Res., 98(B9), 15,937-15,950, doi: 10.1029/93JB00780.

Rice, J. R., N. Lapusta, and K. Ranjith (2001), Rate and state dependent friction and the stability of sliding between elastically deformable solids, Journal of the Mechanics and Physics of Solids, 49(9), 1865 - 1898, doi:http://dx.doi.org/10.1016/S0022-5096(01)00042-4.

Rubin, A. M. (2008), Episodic slow slip events and rate-and-state friction, J. Geophys. Res., 113, B11414, doi:10.1029/1008JB005642.

Scholz, C. H. (1998), Earthquakes and friction laws, Nature, 391 (6662), 37. 
Shampine, L. F., and M. W. Reichelt (1997), The MATLABE ODE suite, SIAM J. Sci. Comput., $18(1), 1-22$.

Tullis, T. E., and J. D. Weeks (1986), Constitutive behavior and stability of frictional sliding of granite, Pure Appl. Geophys, 124(3), 383-414, doi:10.1007/BF00877209.

Zoet, L. K., B. Carpenter, M. Scuderi, R. B. Alley, S. Anandakrishnan, C. Marone, and M. Jackson (2013), The effects of entrained debris on the basal sliding stability of a glacier, Journal of Geophysical Research: Earth Surface, 118(2), 656-666, doi:10.1002/jgrf.20052. 